\title{
Some results on AM-compact operators
}

\author{
Abdelmonaim El Kaddouri Mohammed Moussa
}

\begin{abstract}
We characterize Banach lattices under which each AM-compact (resp. b-AM-compact) operator is Dunford-Pettis. Also, we study the AM-compactness of limited completely continuous operators.
\end{abstract}

\section{Introduction}

Throughout this paper $X, Y$ will denote Banach spaces, and $E, F$ will denote Banach lattices. The positive cone of $E$ will be denoted by $E^{+}$.

The class of AM-compact operators was introduced and studied by DoddsFremlin [9]. We say that an operator $T: E \rightarrow X$ is called AM-compact if the image of each order bounded subset of $E$ is a relatively compact subset of $X$.

Following Aliprantis and Burkinshaw we say that an operator $T: X \rightarrow Y$ is called a Dunford-Pettis operator if for each weakly null sequence $\left(x_{n}\right)$, we have $\left.\lim _{n \rightarrow \infty}\left\|T\left(x_{n}\right)\right\|=0\right)$. Equivalently, $T$ carries relatively weakly compact sets onto relatively compact subsets of $Y[1]$.

Recently, M. Salimi et S. M. Moshtaghioun introduced the class of limited completely continuous operators, and characterized this class of operators and studied some of its properties in [12]. Let us recall that the operator $T: X \longrightarrow Y$ is called limited completely continuous (abb. $l c c)$, if $T$ carries limited and weakly null sequences in $X$ to norm null ones. Alternatively, $T$ is $l c c$ if, and only if, for each limited set $A \subset X$, the set $T(A)$ is relatively compact, [12].

In [5], the authors studied the AM-compactness of Dunford-Pettis operators. Our goal in the first section of this article is to study the Banach lattice under

Received by the editors in January 2013 - In revised form in December 2013.

Communicated by F. Bastin.

2010 Mathematics Subject Classification : 46B42, 47B60, 47B65.

Key words and phrases : AM-compact operator, b-AM-compact operator, limited completely continuous operator, order continuous norm, limited set, Positive Schur property. 
which every AM-compact operator is Dunford-Pettis. In fact,we give necessary conditions under which each AM-compact operator is Dunford-Pettis. More precisely, we show that if any AM-compact operator $T$ from a Banach lattice $E$ such that its norm is order continuous, in a Banach lattice $F$, is Dunford-Pettis then $E$ admits the positive Schur property or the norm of $F$ is order continuous (Theorem 3.4 ). Also, we establish some sufficient conditions for each AM-compact operator is Dunford-Pettis (Theorem 3.2) and with an example, we prove that the condition "the norm of $E$ is order continuous" is essential in Theorem 3.2.

In the second section of this article, we will study the AM-compactness of $l c c$ operators. In fact, we give some sufficient conditions under which each AMcompact operator is an $l c c$ (Theorem 3.6). As a consequence of Theorem 3.2 and Theorem 3.6, we give some sufficient conditions under which each DunfordPettis operator is $l c c$.

\section{Preliminaries}

To state our results, we need to fix some notations and recall some definitions. A Banach lattice is a Banach space $(E,\|\|$.$) such that E$ is a vector lattice and its norm satisfies the following property: for each $x, y \in E$ such that $|x| \leq|y|$, we have $\|x\| \leq\|y\|$. A norm $\|\cdot\|$ of a Banach lattice $E$ is order continuous if for each generalized sequence $\left(x_{\alpha}\right)$ such that $x_{\alpha} \downarrow 0$ in $E,\left(x_{\alpha}\right)$ converges to 0 for the norm $\|\cdot\|$ where the notation $x_{\alpha} \downarrow 0$ means that $\left(x_{\alpha}\right)$ is decreasing, its infimum exists and $\inf \left(x_{\alpha}\right)=0$. Note that if $E$ is a Banach lattice, its topological dual $E^{\prime}$, endowed with the dual norm and the dual order, is also a Banach lattice.

A Banach lattice $E$ is said to have the positive Schur property if every weakly convergent sequence to 0 in $E^{+}$is norm convergent to zero. For example, the Banach space $\ell^{1}$ has the positive Schur property but the Banach space $\ell^{\infty}$ does not has this property.

A Banach lattice $E$ is called a KB-space whenever every increasing norm bounded sequence of $E^{+}$is norm convergent. As an example, each reflexive Banach lattice is a KB-space, but the Banach lattice $c_{0}$ is not a $\mathrm{KB}$-space.

Recall that a subset $A$ of a Banach lattice $E$ is almost order bounded, if for all $\epsilon>0$ there exists $x \in E^{+}$with $A \subset[-x, x]+\epsilon B_{E}$.

A nonzero element $x$ of a vector lattice $E$ is discrete if the order ideal generated by $x$ equals the lattice subspace generated by $x$. The vector lattice $E$ is discrete, if it admits a complete disjoint system of discrete elements. A subset $A$ of a vector lattice $E$ is called order bounded, if it include in an order interval in $E$. A linear mapping $T$ from a vector lattice $E$ into another $F$ is order bounded if it carries order bounded set of $E$ into order bounded set of $F$. We will use the term operator $T: E \longrightarrow F$ between two Banach lattices to mean a bounded linear mapping, it is positive if $T(x) \geq 0$ in $F$ whenever $x \geq 0$ in $E$. The operator $T$ is regular if $T=T_{1}-T_{2}$ where $T_{1}$ and $T_{2}$ are positive operators from $E$ into $F$. Note that each positive linear mapping on a Banach lattice is continuous, If an operator $T: E \longrightarrow F$ between two Banach lattices is positive, then its adjoint $T^{\prime}: F^{\prime} \longrightarrow E^{\prime}$ is likewise positive, where $T^{\prime}$ is defined by $T^{\prime}(f)(x)=f(T(x))$ for each $f \in F^{\prime}$ and for each $x \in E$. For terminologies concerning Banach lattice theory and posi- 
tive operators we refer the reader to the excellent book of Aliprantis-Burkinshaw [1].

\section{Main results}

\subsection{On the classes of Dunford-Pettis and AM-compact operators}

Recall that an operator $T: E \rightarrow Y$ is almost Dunford-Pettis if $\left\|T\left(x_{n}\right)\right\| \rightarrow 0$ for every weakly null sequence $\left(x_{n}\right)$ in $E$ consisting of pairwise disjoint elements [13]. Note that every Dunford-Pettis operator is almost Dunford-Pettis, but the converse is not true in general. Indeed, the identity operator of the Banach lattice $L^{1}[0,1]$ is almost Dunford-Pettis and fails to be Dunford-Pettis. On the other hand, an AM-compact operator is not necessary a Dunford-Pettis. In fact, the identity operator of the Banach lattice $c_{0}$ is AM-compact but fails to be DunfordPettis.

Proposition 3.1. If $T: F \rightarrow X$ is an AM-compact operator and $S: E \rightarrow F$ is an almost Dunford-Pettis operator, then the product $T \circ S$ is Dunford-Pettis.

In the following result, we give a sufficient conditions under which each AM-compact operator is Dunford-Pettis.

Theorem 3.2. Each AM-compact operator $T: E \longrightarrow F$ is Dunford-Pettis if one of the following statements is valid:

1. E has the positive Schur property,

2. F has the Schur property.

Proof. (1) Let $T: E \rightarrow F$ be an operator and $A$ be a relatively weakly compact subset of $E$. Since $E$ has the positive Schur property, it follows from Theorem 3.1 of [8] that $A$ is almost order bounded, then there exists $x \in E^{+}$with $A \subset[-x, x]+\epsilon B_{E}$ and hence $T(A) \subset T([-x, x])+\epsilon\|T\| B_{F}$.

Now, as $T$ is AM-compact then, $T([-x, x])$ is relatively compact in $F$ and hence $T(A)$ is relatively compact. This show that $T$ is Dunford-Pettis.

(2) In this case each operator is Dunford-Pettis.

Recall from [3] that an operator $T: E \rightarrow X$ is said to be b-AM-compact if it carries b-order bounded set of $E$ (i.e., order bounded in $E^{\prime \prime}$ ) into norm relatively compact set of $X$. Note that a b-AM-compact operator is not necessary DunfordPettis. In fact, the identity operator of the Banach lattice $\ell^{2}$ is b-AM-compact (because $\ell^{2}$ is a discrete KB-space) but it is not a Dunford-Pettis operator (because $\ell^{2}$ does not has the Schur property).

As consequence of Theorem 3.2, we obtain the following result, 
Corollary 3.3. Each b-AM-compact operator $T: E \longrightarrow F$ is Dunford-Pettis if one of the following statements is valid:

1. E has the positive Schur property,

2. F has the Schur property.

Reciprocally, we give necessary conditions under which each AM-compact operator is Dunford-Pettis,

Theorem 3.4. Let $E$ and $F$ be two Banach lattices such that the norm of $E$ is order continuous. If each AM-compact operator $T: E \longrightarrow F$ is Dunford-Pettis then one of the following statements is valid:

1. E has the positive Schur property,

2. F has an order continuous norm.

Proof. Assume that $E$ does not has the positive Schur property and that the norm of $F$ is not order continuous. Since $E$ does not have the positive Schur property, it follows from Proposition 2.1 of [2] that there is a disjoint weakly null sequence $\left(x_{n}\right)$ in $E^{+}$with $\left\|x_{n}\right\|=1$ for all $n$. Hence, by Proposition 2.5 of [2], there exists a positive disjoint sequence $\left(g_{n}\right)$ in $E^{\prime}$ with $\left\|g_{n}\right\|=g_{n}\left(x_{n}\right)=1$ for all $n$ and $g_{n}\left(x_{m}\right)=0$ if $n \neq m$. $(\star)$

Since the norm of $E$ is order continuous, it follows from Corollary 2.4.3 of [11] that $g_{n} \rightarrow 0$ for $\sigma\left(E^{\prime}, E\right)$. Hence, the positive operator $Q: E \rightarrow c_{0}$ defined by

$$
Q(x)=\left(g_{n}(x)\right)_{n=1}^{\infty} \text { for all } x \in E,
$$

is well defined. On the other hand, since the norm of $F$ is not order continuous, there exists a disjoint sequence $\left(y_{n}\right)$ of $F^{+}$and $y \in F^{+}$such that $0 \leq y_{n} \leq y$ and $\left\|y_{n}\right\|=1$ for each $n$.

Now, we consider the positive operator $S: c_{0} \rightarrow F$ defined by

$$
S\left(\left(\lambda_{n}\right)\right)=\sum_{n=1}^{\infty} \lambda_{n} y_{n} \text { for all }\left(\lambda_{n}\right) \in c_{0}
$$

The series defining $Q$ is norm convergent for $\left(\lambda_{n}\right) \in c_{0}$ because the sequence $\left(y_{n}\right)$ is disjoint and order bounded.

Now, we consider the positive operator $T=S \circ Q: E \rightarrow c_{0} \rightarrow F$. It is clear that $T$ is AM-compact but $T$ is not Dunford-Pettis. In fact, note that $\left(x_{n}\right)$ is a weakly null sequence of $E^{+}$and then by $(\star)$ we have

$$
T\left(x_{n}\right)=S \circ Q\left(x_{n}\right)=S\left(e_{n}\right)=y_{n} \text { for all } n .
$$

If $T$ is Dunford-Pettis, then $\lim _{n \rightarrow \infty}\left\|T\left(x_{n}\right)\right\|=\lim _{n \rightarrow \infty}\left\|y_{n}\right\|=0$, which contradicts with $\left\|y_{n}\right\|=1$ for all $n$.

Remark 1. The assumption "E has an order continuous norm" is essential in Theorem 3.4. In fact, each operator $T: \ell^{\infty} \rightarrow c$ is Dunford-Pettis but neither $\ell^{\infty}$ has the positive Schur property nor $c$ has an order continuous norm. 


\subsection{On the classes of limited completely continuous and AM-compact operators}

To study the AM-compactness of $l c c$ operators, we need to recall some definitions.

A norm bounded subset $A$ of $X$ is said limited set if every weak ${ }^{\star}$ null sequence $\left(f_{n}\right)$ of $X^{\prime}$ converges uniformly to zero on $A$ [7], that is, $\lim _{n \rightarrow \infty} \sup _{x \in A}\left|\left\langle f_{n}, x\right\rangle\right|=0$.

Note that every relatively compact set is limited but the converse is not true in general. Indeed, the set $\left\{e_{n}: n \in \mathbb{N}\right\}$ of unit coordinate vectors is a limited set in $\ell^{\infty}$ which is not relatively compact. If every limited subset of $X$ is relatively compact then, $X$ has the Gelfand-Phillips property (abb. GP-property). Alternatively, $X$ has the GP-property if, and only if, every limited and weakly null sequence $\left(x_{n}\right)$ in $X$ is norm null ones [10]. As an example, the classical Banach spaces $c_{0}$ and $\ell^{1}$ has the GP-property but the Banach space $\ell^{\infty}$ does not has this property.

Let us recall from Borwein [6] that, $X$ has the Dunford-Pettis* property (abb. $\mathrm{DP}^{\star}$ property) if every relatively weakly compact subset of $X$ is limited. Also, the lattice operations in $E^{\prime}$ are called weak ${ }^{\star}$ sequentially continuous if the sequence $\left(\left|f_{n}\right|\right)$ converges to 0 for the weak ${ }^{\star}$ topology $\sigma\left(E^{\prime}, E\right)$ whenever the sequence $\left(f_{n}\right)$ converges to 0 for the topology $\sigma\left(E^{\prime}, E\right)$. And, the lattice operations of $E$ are weak sequentially continuous if the sequence $\left(\left|x_{n}\right|\right)$ converges to 0 for the weak topology $\sigma\left(E, E^{\prime}\right)$ whenever the sequence $\left(x_{n}\right)$ converges to 0 for the topology $\sigma\left(E, E^{\prime}\right)$.

Note that there exists a lcc operator which is not AM-compact. Indeed, the identity operator of the Banach space $L^{2}[0,1]$ is $l c c$ (because $L^{2}[0,1]$ has the GP-property) but fails to be AM-compact (because $L^{2}[0,1]$ is not discrete).

To establish our first result in this section, we will need the following Lemma, which gives a characterization of limited order intervals .

Lemma 3.5. Let E be a Banach lattice. Then the following assertions are equivalent

1. E' has weak ${ }^{\star}$ sequentially continuous lattice operations,

2. for each $x \in E^{+}$, the order interval $[-x, x]$ is limited.

Proof. $[-x, x]$ is limited if, and only if, $\sup \left\{\left|f_{n}(z)\right| ; z \in[-x, x]\right\} \longrightarrow 0$.

As $\left|f_{n}\right|(x)=\sup \left\{\left|f_{n}(z)\right| ; z \in[-x, x]\right\}$, we conclude that $\left|f_{n}\right|$ converge weak ${ }^{\star}$ to 0 , i.e. $E^{\prime}$ has weak ${ }^{\star}$ sequentially continuous lattice operations.

The following result gives some sufficient conditions under which each $l c c$ operator from $E$ into $X$ is AM-compact,

Theorem 3.6. Each lcc operator $T: E \rightarrow X$ is AM-compact if one of the following assertions is valid:

1. E has an order continuous norm and has the $D P^{\star}$ property,

2. $E^{\prime}$ has weak ${ }^{\star}$ sequentially continuous lattice operations,

3. $F$ is a discrete with an order continuous norm, 
Proof. (1) Let $x \in E^{+}$, since $E$ has an order continuous norm then, the order interval $[-x, x]$ is weakly relatively compact.

On the other hand, since $E$ has the $\mathrm{DP}^{\star}$ property then, $[-x, x]$ is a limited subset of $X$. Now, since $T$ is $l c c$ then $T([-x, x])$ is relatively compact and hence $T$ is AM-compact.

(2) Let $x \in E^{+}$and $E^{\prime}$ has weak ${ }^{\star}$ sequentially continuous lattice operations then, it follows from Lemma 3.5 that the order interval is a limited subset of $E$. Now, since $T$ is $l c c$ then $T([-x, x])$ is relatively compact and hence $T$ is AM-compact.

(3) In this case, each operator $T: E \rightarrow X$ is AM-compact.

Note that each Dunford-Pettis operator is $l c c$, but the converse is not true in general. Indeed, the identity operator of the Banach lattice $\ell^{2}$ is $l c c$ but fails to be a Dunford-Pettis operator. However, as a consequence of Theorem 3.2 and Theorem 3.6, we obtain sufficient conditions under which each Dunford-Pettis operator is $l c c$,

Corollary 3.7. Let $E$ and $F$ two Banach lattices. Then, each lcc operator $T: E \rightarrow X$ is Dunford-Pettis if one of the following conditions is valid:

1. E admits the positive Shur property and has the $D P^{\star}$ property,

2. E admits the positive Shur property and $E^{\prime}$ has a sequentially continuous lattice operations,

3. E admits the positive Shur property and $F$ is discreet with order continuous norm.

The following Proposition gives some properties whenever each $l c c$ operator from a Dedekind $\sigma$-complete Banach lattice $E$ into $F$ is AM-compact,

Proposition 3.8. Let $E$ and $F$ be a two Banach lattices such that $E$ is Dedekind $\sigma$-complete and the lattice operations of $F$ are weakly sequentially continuous. If each lcc operator $T: E \rightarrow X$ is AM-compact then one of the following assertions is valid:

1. E has an order continuous norm,

2. $F$ is a discrete with an order continuous norm.

Proof. Assume that the norm of $E$ is not order continuous and that $F$ is not discrete with order continuous norm.

Since $E$ is Dedekind $\sigma$-complete, it follows from Corollary 2.4.3 of [11] that $E$ contains a sub-lattice which is isomorphic to $\ell^{\infty}$ and there exists a positive projection $P: E \rightarrow \ell^{\infty}$.

On the other hand, as the lattice operations of $F$ are weakly sequentially continuous and $F$ is not discrete with order continuous norm, it follows from Theorem 3.7 [4] that there exists a regular Dunford-Pettis operator $S: \ell^{\infty} \rightarrow F$ which is not AM-compact.

Since $S: \ell^{\infty} \rightarrow F$ is Dunford-Pettis, then it is order weakly compact and and Since $\ell^{\infty}$ is an AM-space with unit, then $S: \ell^{\infty} \rightarrow F$ is weakly compact. It follows from Corollary 2.5 of [12] that the operator $S$ is $l c c$. 
Now we consider the operator product $T=S \circ P: E \rightarrow F$. Since the operator $S$ is $l c c$ then operator $T$ is $l c c$ because the class of $l c c$ operators is a two-sided ideal. But it is not AM-compact. Otherwise, the operator $T \circ \imath=S$ would be AM-compact ( where $\imath: \ell^{\infty} \rightarrow F$ is the natural embedding). This presents a contradiction.

As consequence of Proposition 3.8, we have the following result,

Corollary 3.9. Let $F$ be a Banach lattice with weakly sequentially continuous lattice operations. Then the following assertions are equivalent:

1. each lcc operator $T: \ell^{\infty} \rightarrow F$ is AM-compact,

2. the norm of $F$ is order continuous.

\section{References}

[1] C.D. Aliprantis and O. Burkinshaw, Positive Operators, Reprint of the 1985 original, Springer, Dordrecht, 2006.

[2] B. Aqzzouz, A. Elbour and A. W. Wickstead, Positive almost Dunford-Pettis operators and their duality, Positivity, DOI: 10.1007/s11117-010-0050-3.

[3] B. Aqzzouz and J. Hmichane, The class of b-AM-compact operators, Quaestions Mathematicae, Volume 36, Issue 3, 2013, DOI:10.2989/16073606.2013.805869.

[4] B. Aqzzouz and J. Hmichane, AM-compactness of some classes of operators, Comment.Math.Univ.Carolin, 53,4 (2012) 509-518.

[5] B. Aqzzouz and L. Zraoula, AM-compactness of positive Dunford-Pettis operators on Banach lattices, Rend. Circ. Mat. Palermo, (2) 56 (2007), no. 3, 305-316.

[6] J. Borwein, M. Fabian and J. Vanderwerff, Characterizations of Banach spaces via convex and other locally Lipschitz functions, Acta. Math. Vietnam., 22, no. 1, 1997, 53-69.

[7] J. Bourgain and J. Diestel, Limited operators and strict cosingularity, Math. Nachr. 119 (1984), 55-58.

[8] Z.L. Chen and A.W. Wickstead, L-weakly and M-weakly compact operators[J], Indag. Mathem., N. S., 10(3), 321-336 (1999).

[9] P.G. Dodds and D.H. Fremlin, Compact operators on Banach lattices, Israel J. Math, 34 (1979) 287-320.

[10] L. Drewnowski, On Banach spaces with the GelfandPhillips property, Math. Z. 193 (1986), 405411. 
[11] P. Meyer-Nieberg, Banach lattices, Universitext. Springer-Verlag, Berlin, 1991.

[12] M. Salimi and S. M. Moshtaghioun, The Gelfand-Phillips property in closed subspaces of some operator spaces, Banach Journal of Mathematical Analysis, vol. 5, no. 2, pp. 84-92, 2011.

[13] W. Wnuk, Banach lattices with the weak Dunford-Pettis property, Atti Sem. Mat. Fis. Univ. Modena 42(1), 227-236 (1994). MR 1282338 (95g:46034)

Université Ibn Tofail,

Faculté des Sciences, Département de Mathématiques,

B.P. 133, Kénitra, Morocco.

email : elkaddouri.abdelmonaim@gmail.com 